\title{
Sildenafil citrate as an adjuvant to clomiphene citrate for ovulation induction in polycystic ovary syndrome: crossover randomized controlled trial
}

\author{
Walid E. Mohammed ${ }^{1}$, Mustafa M. Abbas ${ }^{1}$, Ibrahim A. Abdelazim ${ }^{1,2}$, Mohammed M. Salman ${ }^{1}$ \\ 'Department of Obstetrics and Gynaecology, Faculty of Medicine, Ain Shams University, Cairo, Egypt \\ ${ }^{2}$ Department of Obstetrics and Gynaecology, Ahmadi Hospital, Kuwait Oil Company (KOC), Kuwait
}

\begin{abstract}
Introduction: To evaluate sildenafil citrate as an adjuvant to clomiphene citrate (CC) for ovulation induction (OI) in women with polycystic ovary syndrome (PCOS).

Material and methods: A total of 595 infertile PCOS women were randomly assigned into either clomiphene/sildenafil (C/S) group or CC group. Transvaginal (TVS)-Doppler studies were done for participants when the dominant follicle reach $16 \mathrm{~mm}$, to measure the resistance index, pulsatility index, and maximum velocity of sub-endometrial, uterine, and ovarian vessels. Participants were examined using TVS on the $21^{\text {st }}$ day of the cycle to detect ovulation or, after a positive pregnancy test, for documentation of pregnancy. Participants with negative pregnancy tests were given 2 months' rest without Ol, followed by crossover of Ol medication between the 2 studied groups. The crossover results were assessed by TVS, TVS-Doppler, and pregnancy test.

Results: The endometrial thickness was significantly higher among the C/S than the CC group during the first 3 months ( $9.6 \pm 1.2$ vs. $8.7 \pm 1.0 \mathrm{~mm}$, respectively, $p=0.003)$ and after crossover of Ol $(9.1 \pm 1.3$ vs. $8.2 \pm 1.0 \mathrm{~mm}$, respectively, $p=0.007)$.

The chemical and clinical pregnancy rates were significantly higher among the C/S compared to the CC group (39.8\% and $36.6 \%$ vs. $25 \%$ and $18.98 \%$, respectively) ( $p=0.01$ and 0.001 , respectively) during the first 3 months and after crossover of OI ( $36.6 \%$ and $33.1 \%$ vs. $23.8 \%$ and $20.6 \%$, respectively) ( $p=0.02$ and 0.01 , respectively).

Conclusions: SC as an adjuvant to CC for OI in PCOS women increases the chemical and clinical pregnancy rates. It also improves the endometrial thickness and ovulation rate through improved endometrial and ovarian Doppler indices.
\end{abstract}

Key words: induction of ovulation, clomiphene citrate, sildenafil citrate, PCOS.

\section{Introduction}

Anovulation accounts for about $20 \%$ of infertility [1]. Around $85 \%$ of anovulatory infertilities have normal gonadotropin levels and are classified as type 2 anovulation according to World Health Organization classification [2]. Most of the anovulation is due to polycystic ovary syndrome (PCOS) [3].

Guidelines recommend clomiphene citrate (CC) as first-line ovulation induction (OI) in women with type 2 anovulation or PCOS [2, 3].

Clomiphene citrate is an anti-oestrogen, non-steroidal compound, which blocks oestrogen receptors at the hypothalamus, releasing it from negative feedback, and augmenting $\mathrm{GnRH}$ release. Subsequently, the pituitary production of gonadotropins increased, resulting in follicular growth and ovulation [4].

The ovulation and pregnancy rates are $70-85 \%$ and $40-70 \%$, respectively, when CC is used for $\mathrm{Ol}$ [5].
About $15 \%$ of anovulatory women do not respond to CC (CC resistance) [3]. Failure to achieve pregnancy despite achieving ovulation after CC treatment is defined as CC failure $[2,4]$.

The anti-oestrogenic effect of CC on the endometrium is an important cause of CC failure [6]. Oestrogen is necessary for endometrial development during the follicular phase of the menstrual cycle [7].

The anti-oestrogenic effect of CC impairs endometrial development, and endometrial biopsies from women on $\mathrm{CC}$ show decreased number and diameter of endometrial glands, with delayed glandular maturation [8].

The poor endometrial development appears by ultrasound as thin endometrium. The oestrogen-mediated development of the endometrium is dependent on endometrial blood flow. Vasodilators were used previously as a potential solution to improve endometrial blood flow and thickness in infertile women with CC 
failure [9]. Sildenafil citrate (SC) inhibits phosphodiesterase-5 (PDE5) and CGMP-dependent protein kinases (PKGs), and maximizes the effect of nitric oxide (NO), thus facilitating smooth muscle cell relaxation [10]. Sildenafil citrate could improve uterine blood flow and induce endometrium proliferation [11]. Therefore, this study was designed to evaluate SC as an adjuvant to CC for Ol in PCOS women.

\section{Material and methods}

A total of 595 infertile PCOS women were recruited for this crossover randomized controlled study, which was conducted from January 2018 to December 2019 after approval by the institutional ethical committee and after informed consents in accordance with the Helsinki declaration.

The study was completed with the final analysis of the data of 432 PCOS women, [163 were excluded because of non-matched inclusion criteria (141) and refusal to participate (22)].

Inclusion criteria include infertile PCOS women (according to Rotterdam's Criteria), aged 21-35 years, non-smokers, without other causes of infertility, and no contraindications for SC.

Women with congenital uterine anomalies, fibroid, adenomyosis, endometrial polyp(s), Asherman's syndrome, chronic use of non-steroidal anti-inflammatory (NSAIDs), causes of infertility other than PCOS, and contraindications of sildenafil were excluded from this study.

The European Society for Human Reproduction/ American Society of Reproductive Medicine (ESHRE/ ASRM) criteria were used to diagnose PCOS [12]. The polycystic ovaries were diagnosed by the threshold for polycystic ovary morphology $\geq 20$ follicles per ovary and/or an ovarian volume $\geq 10 \mathrm{~mL}$ on either ovary, ensuring that there are no corpora lutea, cysts, or dominant follicles [13].

According to the ESHRE/ASRM recommendation, causes of hyperandrogenism such as Cushing`s syndrome, late-onset congenital adrenal hyperplasia, and androgen-secreting tumours were excluded before diagnosing PCOS [14-16].

The contraindications of SC include sickle cell anaemia, uncontrolled either high or low blood pressure, cardiac women (i.e. hypertrophic cardiomyopathy, mitral or aortic stenosis, and chronic heart failure), and chronic liver disease.

Participants were randomly assigned to 2 groups (216 women in each group): clomiphene/sildenafil (C/S) group and CC group.

Participants in the C/S group received CC $100 \mathrm{mg} /$ daily from the $2^{\text {nd }}$ to $6^{\text {th }}$ day of the menstrual cycle for OI plus oral SC $20 \mathrm{mg} 12$-hourly for 10 days starting from the $2^{\text {nd }}$ day of cycle (as an adjuvant to CC).
Participants in the CC group received CC $100 \mathrm{mg} /$ daily from the $2^{\text {nd }}$ to $6^{\text {th }}$ day of the cycle for OI.

Participants were subjected to transvaginal sonography (TVS) on the $9^{\text {th }}$ and $13^{\text {th }}$ day of the menstrual cycle, and when the dominant follicle reach $16 \mathrm{~mm}$ diameter the resistance index $(\mathrm{RI})$, pulsatility index $(\mathrm{PI})$, and maximum velocity (T-max) of sub-endometrial, uterine, and ovarian vessels were measured using TVS-Doppler.

Participants were examined using TVS on the $21^{\text {st }}$ day of the cycle for detection of ovulation and after a positive pregnancy test for documentation of clinical pregnancy.

Participants with negative pregnancy tests were given 2 months rest without Ol, followed by the crossover of Ol medication between the 2 studied groups ( 145 women shifted to C/S protocol, and 126 women to CC protocol).

Crossover OI means participants in the $\mathrm{C} / \mathrm{S}$ group without any documented ovulation after 3 cycles of OI received the CC protocol of $\mathrm{OI}$, and participants in the CC group without any documented ovulation after 3 cycles of OI received the C/S protocol of OI. The crossover results were assessed by TVS, TVS-Doppler, and pregnancy test (as described previously).

The side effects related to SC or CC were assessed using an objective scaling scoring system: 0 (no side effects), 1 (mild side effects, without affecting lifestyle), 2 (severe side effects, but controlled by another method), 3 (severe, non-controlled side effects and participant still agreeing to continue medication), 4 (severe, non-controlled and participant not agreeing to continue medication). Collected data were analysed to evaluate SC as an adjuvant to CC for OI in PCOS women.

\section{Sample size justification}

The required sample size for this study was calculated using G Power software version 3.1.9.7 for sample size calculation, setting the $\alpha$-error probability at 0.05 , power (1- $\beta$ error probability) at $0.95 \%$, and effective sample size $(w)$ at 0.5 . An effective sample $\geq 210$ PCOS-women in 2 studied groups was needed to produce a statistically acceptable figure.

\section{Statistical analysis}

Collected data statistically analysed using Statistical Package for Social Sciences (SPSS) version 20 (Chicago, IL, USA). Numerical variables were presented as mean and standard deviation $( \pm S D)$, while categorical variables were presented as number (n) and percentage (\%). The chi-square test $\left(\chi^{2}\right)$ and Student's t-test were used for the analysis of qualitative and quantitative variables, respectively. $p<0.05$ was considered significant. 
Table 1. Follicular maturation, ovulation, endometrial thickness, and chemical and clinical pregnancies of the 2 studied groups in the first 3 months

\begin{tabular}{|c|c|c|c|}
\hline Variables & $\begin{array}{l}\text { C/S group } \\
n=216(\%)\end{array}$ & $\begin{array}{c}\text { CC group } \\
n=216(\%)\end{array}$ & $\begin{array}{l}p \text {-value } \\
(95 \% \mathrm{Cl})\end{array}$ \\
\hline Follicular Maturation & $107(49.5)$ & $74(34.3)$ & $0.03^{\star}$ \\
\hline Ovulation & $99(45.8)$ & $63(29.2)$ & $0.01^{\star}$ \\
\hline Endometrial thickness [mm] & $9.6 \pm 1.2$ & $8.7 \pm 1.0$ & $0.003^{*}(0.69,0.9,1.11)$ \\
\hline \multicolumn{3}{|l|}{ Chemical pregnancy } & \multirow{3}{*}{$0.01^{\star}$} \\
\hline Positive & $86(39.8)$ & $54(25)$ & \\
\hline Negative & $130(60.2)$ & $162(75)$ & \\
\hline \multicolumn{3}{|l|}{ Clinical pregnancy } & \multirow{3}{*}{$0.001^{*}$} \\
\hline Positive & $79(36.6)$ & $41(18.98)$ & \\
\hline Negative & $137(63.4)$ & $175(81.02)$ & \\
\hline
\end{tabular}

*Significant

CC group - clomiphene citrate group, $\mathrm{Cl}$ - confidence interval, C/S group - clomiphene/sildenafil group

Data presented as number and percentage (\%) and mean \pm SD (standard deviation).

Independent t-test used for statistical analysis when data presented as mean $\pm \mathrm{SD}$.

Chi-square $\left(\chi^{2}\right)$ test used for statistical analysis when data presented as number and \%.

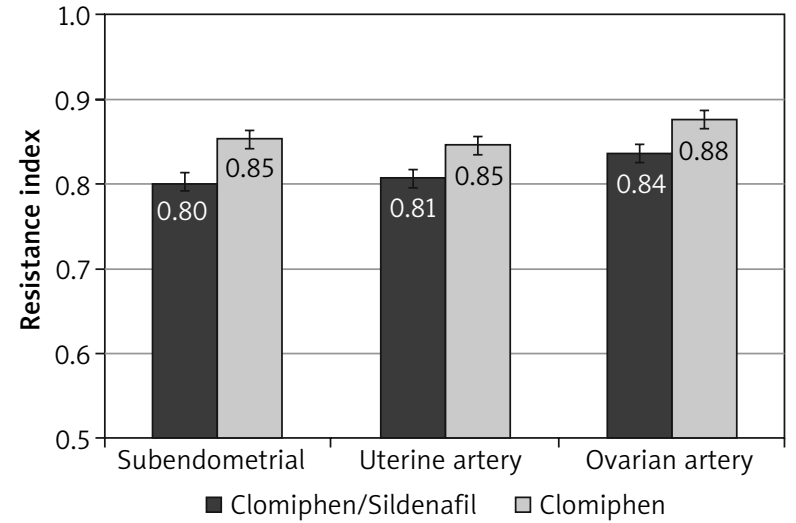

Fig. 1. The mean resistance index of the 2 studied groups in the first 3 months

\section{Results}

During the first 3 months, the follicular maturation and ovulation were significantly higher among $\mathrm{C} / \mathrm{S}$ (49.5\% and $45.8 \%$, respectively) compared to the CC group (34.3\% and $29.2 \%$, respectively) ( $p=0.03$ and 0.01 , respectively). The endometrial thickness was sig-

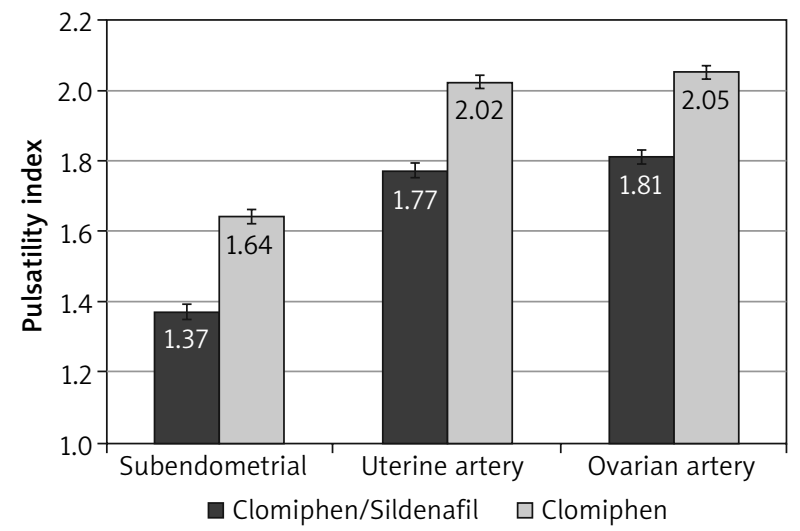

Fig. 2. The mean pulsatility index of the 2 studied groups in the first 3 months nificantly higher among $\mathrm{C} / \mathrm{S}$ compared to the CC group (9.6 \pm 1.2 vs. $8.7 \pm 1.0 \mathrm{~mm}$, respectively, $p=0.003$ ). The chemical and clinical pregnancy rates were significantly higher among C/S compared to the CC group (39.8\% and $36.6 \%$ vs. $25 \%$ and $18.98 \%$, respectively) ( $p=0.01$ and 0.001 , respectively) (Table 1 ).

During the first 3 months, the mean RI was significantly lower among the $\mathrm{C} / \mathrm{S}$ group compared to the CC group for sub-endometrial arteries (0.8 vs. 0.85 , respectively), uterine artery ( 0.81 vs. 0.85 , respectively), and ovarian artery (0.84 vs. 0.88 , respectively, $p<0.001$ ) (Fig. 1). The mean PI was also significantly lower among the C/S group compared to the CC group for sub-endometrial arteries (1.37 vs. 1.64, respectively), uterine artery (1.77 vs. 2.02, respectively), and ovarian artery (1.81 vs. 2.05, respectively, $p<0.001$ ) (Fig. 2). The mean T-max was significantly higher among the $\mathrm{C} / \mathrm{S}$ group compared to the CC group for sub-endometrial arteries (10 vs. $9.6 \mathrm{~cm} / \mathrm{s}$, respectively), uterine artery (10.8 vs. $10.4 \mathrm{~cm} / \mathrm{s}$, respectively), and ovarian artery (9.3 vs. $9.2 \mathrm{~cm} / \mathrm{s}$, respectively, $p<0.001$ ) (Fig. 3).

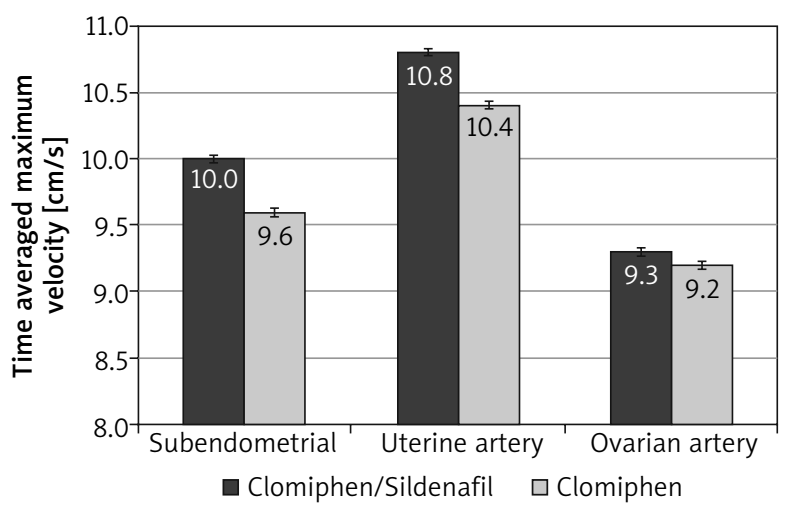

Fig. 3. The mean time-averaged maximum velocity of the 2 studied groups in the first 3 month 
Table 2. Follicular maturation, ovulation, endometrial thickness, and chemical and clinical pregnancies of the 2 studied groups after crossover of ovulation induction medication

\begin{tabular}{|c|c|c|c|}
\hline Variables & $\begin{array}{c}\text { C/S group } \\
n=216(\%)\end{array}$ & $\begin{array}{c}\text { CC group } \\
n=216(\%)\end{array}$ & $\begin{array}{l}p \text {-value } \\
(95 \% \mathrm{Cl})\end{array}$ \\
\hline Follicular Maturation & $63(43.4)$ & $38(30.2)$ & $0.02^{*}$ \\
\hline Ovulation & $60(41.4)$ & $36(28.6)$ & $0.02^{*}$ \\
\hline Endometrial thickness [mm] & $9.1 \pm 1.3$ & $8.2 \pm 1.1$ & $0.007^{\star}(-0.33,-0.1,0.13)$ \\
\hline \multicolumn{3}{|l|}{ Chemical pregnancy } & \multirow{3}{*}{$0.02^{*}$} \\
\hline Positive & $53(36.6)$ & $30(23.8)$ & \\
\hline Negative & $92(63.4)$ & $96(76.2)$ & \\
\hline \multicolumn{3}{|l|}{ Clinical pregnancy } & \multirow{3}{*}{$0.01^{*}$} \\
\hline Positive & $48(33.1)$ & $26(20.6)$ & \\
\hline Negative & 97 (66.9) & $100(79.4)$ & \\
\hline
\end{tabular}

*Significant

CC group - clomiphene citrate group, $\mathrm{Cl}$ - confidence interval, $\mathrm{C} / \mathrm{S}$ group - clomiphene/sildenafil group

Data presented as number and percentage (\%) and mean \pm SD (standard deviation).

Independent t-test used for statistical analysis when data presented as mean \pm SD.

Chi-square $\left(\chi^{2}\right)$ test used for statistical analysis when data presented as number and percentage.

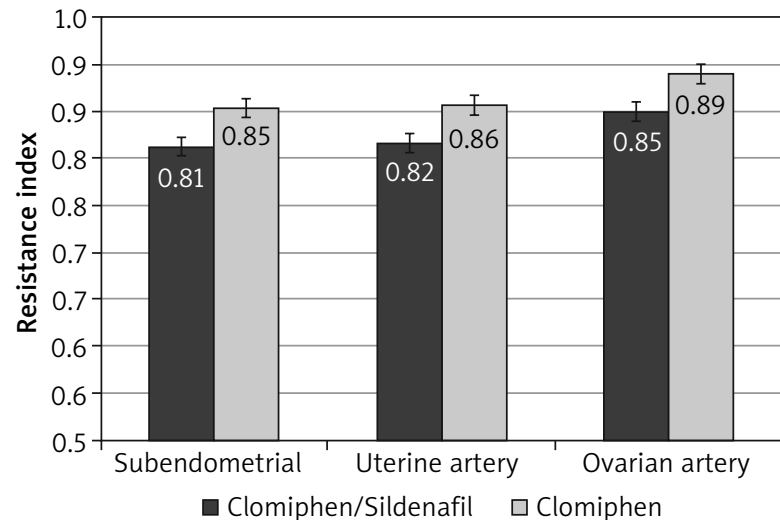

Fig. 4. The mean resistance index of the 2 studied groups after crossover of ovulation induction medications

After the crossover of Ol medication, the follicular maturation and ovulation were significantly higher among the C/S group ( $43.4 \%$ and $41.4 \%$, respectively) compared to the CC group $(30.2 \%$ and $28.6 \%$, respectively) ( $p=0.02$ and 0.02 , respectively). The endometrial thickness was significantly higher among the C/S group compared to the CC group $(9.1 \pm 1.3$ vs. $8.2 \pm 1.0 \mathrm{~mm}$, respectively, $p=0.007)$. The chemical and clinical pregnancy rates were significantly higher among the $\mathrm{C} / \mathrm{S}$ group compared to the CC group (36.6\% and $33.1 \%$ vs. $23.8 \%$ and $20.6 \%$, respectively) ( $p=0.02$ and 0.01 , respectively) (Table 2).

After the crossover of OI medications, the mean $\mathrm{RI}$ was significantly lower among the C/S group compared to the CC group for sub-endometrial arteries (0.81 vs. 0.85 , respectively), uterine artery ( 0.82 vs. 0.86 , respectively), and ovarian artery (0.85 vs. 0.89 , respectively, $p<0.001$ ) (Fig. 4). The mean PI was also significantly lower among the C/S group compared to the CC group for sub-endometrial arteries (1.53 vs. 1.73, respectively), uterine artery (1.66 vs. 1.88, respectively), and ovar-

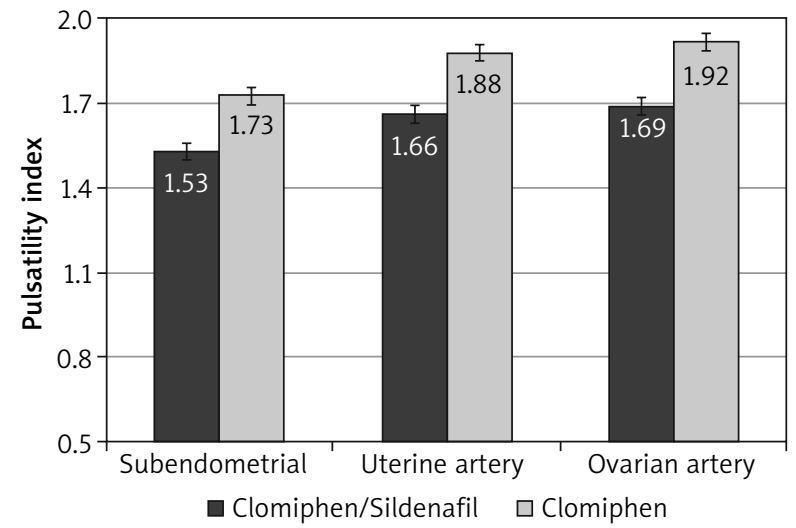

Fig. 5. The mean pulsatility index of the 2 studied groups after crossover of ovulation induction medications

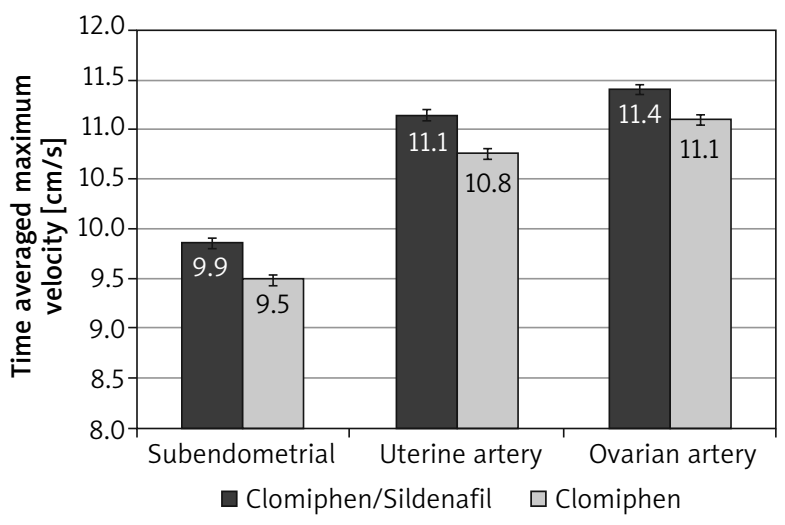

Fig. 6. The mean time-averaged maximum velocity of the 2 studied groups after crossover of ovulation induction medications

ian artery (1.69 vs. 1.92, respectively, $p<0.001$ ) (Fig. 5). The mean T-max was significantly higher among the $\mathrm{C} / \mathrm{S}$ group compared to the CC group for sub-endometrial arteries ( $9.9 \mathrm{vs} .9 .5 \mathrm{~cm} / \mathrm{s}$, respectively), uterine artery (11.1 vs. $10.8 \mathrm{~cm} / \mathrm{s}$, respectively), and ovarian artery (11.4 vs. $11.1 \mathrm{~cm} / \mathrm{s}$, respectively, $p<0.001$ ) (Fig. 6). 
Table 3 . The side effects reported in the 2 studied groups

\begin{tabular}{lccc}
\hline Variables & $\begin{array}{c}\text { C/S group } \\
n=216 \\
\text { women (\%) }\end{array}$ & $\begin{array}{c}\text { CC group } \\
n=216 \\
\text { women (\%) }\end{array}$ & p-value \\
\hline Headache & $106(49.1)$ & $51(23.6)$ & $0.0001^{*}$ \\
\hline Tachycardia & $28(12.96)$ & $9(4.16)$ & $0.002^{\star}$ \\
\hline Hypotension & $12(5.6)$ & $3(1.38)$ & $0.01^{*}$
\end{tabular}

CC group - clomiphene citrate group, C/S group - clomiphene/sildenafil group

*significant

Data presented as number and percentage.

Chi-square $\left(\chi^{2}\right)$ test used for statistical analysis.

The side effects reported in this study were mild (score 1, not affecting lifestyle) and were significantly more frequent among the $\mathrm{C} / \mathrm{S}$ group compared to the CC group. Headache was the commonest side effect (49.1\% vs. $23.6 \%$, respectively, $p=0.0001)$ followed by tachycardia ( $12.96 \%$ vs. $4.16 \%$, respectively, $p=0.002$ ), and hypotension ( $5.6 \%$ vs. $1.38 \%$, respectively, $p=0.01$ ) (Table 3).

\section{Discussion}

A total of 595 infertile PCOS women were recruited for this crossover randomized controlled study, and it was completed with the final analysis of the data of 432 PCOS women.

Participants were randomly assigned into 2 groups: the C/S group (who received CC for Ol plus SC as an adjuvant to $\mathrm{CC}$ ) and the $\mathrm{CC}$ group (who received CC for $\mathrm{OI})$. The collected data were analysed to evaluate SC as an adjuvant to CC for OI in PCOS women.

The chemical and clinical pregnancy rates were significantly higher among C/S compared to the CC group ( $p=0.01$ and 0.001 , respectively) during first the 3 months and after the crossover of OI medications ( $p=0.02$ and 0.01 , respectively).

Fahmy et al. compared the use of SC as an adjuvant to CC during OI, and found the use of SC as an adjuvant to CC during Ol improved the endometrial thickness, follicular number, and pregnancy rate [17].

Mohamed, compared the use of SC as an adjuvant to letrozole for OI in PCOS and found the endometrial thickness and clinical pregnancy rate were significantly higher among the SC/letrozole group compared to letrozole alone (12.7 $\mathrm{mm}$ and 55\% vs. $9.8 \mathrm{~mm}$ and 15\%, respectively) [18].

In addition, Mohamed, found the pregnancy risk ratio was 3.7 higher in the SC/letrozole group compared to the letrozole alone [18].

However, Check et al. found that neither vaginal oestrogen nor sildenafil significantly improved the endometrial thickness or endometrial blood flow in frozen embryo transfer cycles [19].

The endometrial thickness in this study was significantly higher among the C/S group compared to the $C C$ group during the first 3 months $(p=0.003)$ and after the crossover of $\mathrm{OI}$ medication $(p=0.007)$.
Paulus et al. also reported that the use of SC during ART improves the uterine artery blood flow and endometrial thickness in women with thin endometria [20].

Moreover, Fisch et al. studied the effect of SC on endometrial thickness in 148 couples with unexplained infertility and found that SC significantly increases endometrial thickness when used from day 8 to day 13 of the cycle [21].

The effect of SC on endometrial thickness using different routes has been reported. Sher and Fisch, evaluated in a preliminary cross-over study the effect of Vaginal Sildenafil (VS) on the in vitro fertilization (IVF) outcome after multiple IVF failures attributed to poor endometrial thickness in women with normal ovarian reserve and at least 2 prior IVF cycles [22]. Sher and Fisch, found that VS improved the endometrial development in $70 \%$ of studied women [22].

Takasaki et al. examined whether thin endometrium can be improved by increasing uterine radial artery blood flow using different vasodilators, and they found significant improvement of endometrial thickness and uterine radial artery $\mathrm{Rl}$ after the use of vasodilators in women with thin endometria [23].

Thus, the use of sildenafil cannot be expected to help all women with thin endometria. Women with intractable damage of basal endometria may be less likely to respond to increased uterine blood flow caused by sildenafil. In addition, the effect of sildenafil on the endometrium requires an adequate oestrogen level [24].

The mean RI and PI of sub-endometrial, uterine, and ovarian arteries was significantly lower among C/S compared to the CC group during the first 3 months, and after the crossover of Ol medications in this study.

Das et al. and Malinova et al. investigated the role of combined VS and CC vs. CC alone on endometrial thickness, endometrial volume, and endometrial Doppler indices on the day of hCG, during intrauterine insemination (IUI), and found that the use of VS as an adjuvant to CC during Ol achieved significantly higher endometrial thickness, with reduced uterine artery vascular resistance and increased pregnancy rate $[25,26]$.

Ashoush and Abdelshafy, studied SC as an adjuvant to CC for OI in PCOS women with CC failure and found that the use of SC as an adjuvant to CC for OI after CC failure was associated with significantly higher clinical pregnancy rates, endometrial thickness, and improved sub-endometrial blood flow indices [27].

Li et al. in a meta-analysis, studied the effect of SC on infertile women with thin endometria and found that the endometrial thickness and pregnancy rate were significantly higher in the SC group than controls [28]. In addition, they found that the radial artery RI was significantly lower in the SC group than controls [28].

Li et al. concluded that SC improves the endometrial thickness and pregnancy rate in infertile women with thin endometria [28]. 
A Cochrane review found the vasodilators had a beneficial effect on endometrial thickness and slightly improved the clinical pregnancy rate (RR 1.45; 95\% CI: 1.19-1.77) [29].

The side effects reported in this study were mild (score 1) and were significantly more frequent among the $\mathrm{C} / \mathrm{S}$ group compared to the CC group. Headache was the commonest side effect $(p=0.0001)$ followed by tachycardia $(p=0.002)$ and hypotension $(p=0.01)$.

Fahmy et al. reported headache as the most common side effect with SC (20\%), followed by flushing (11.4\%), blurring of vision (5.7\%), and gastrointestinal disorders (5.7\%) [17]. The side effects reported with SC are usually mild to moderate, dose-related, and include headache, flushing, blurring of vision, nausea, and dyspepsia [30-31].

This study found that $\mathrm{SC}$ as an adjuvant to CC for Ol in PCOS women increases the chemical and clinical pregnancy rates. It also improves the endometrial thickness and ovulation rate through improved endometrial and ovarian Doppler indices.

Women who refused to participate and/or give consent was the only limitation faced during this study. Further, larger studies are needed to confirm the beneficial effect of sildenafil when used by different routes (oral or vaginal) on the endometrial pattern, ovulation, and pregnancy rates.

\section{Conclusions}

Sildenafil citrate as an adjuvant to CC for OI in PCOS women increases the chemical and clinical pregnancy rates. It also improves the endometrial thickness and ovulation rate through improved endometrial and ovarian Doppler indices.

\section{Acknowledgement}

The studied women were included in this study after informed consents in accordance with the Helsinki declaration and according to the hospital protocol.

\section{Disclosure}

The authors report no conflicts of interest.

\section{References}

1. Weiss NS, van Vliet MN, Limpens J, et al. Endometrial thickness in women undergoing IUI with ovarian stimulation. How thick is too thin? A systematic review and meta-analysis. Hum Reprod 2017; 32: 1009-1018.

2. Fauser BC, Tarlatzis BC, Rebar RW, et al. Consensus on women's health aspects of Polycystic Ovary Syndrome (PCOS): the Amsterdam ESHRE/ ASRM-Sponsored $3^{\text {rd }}$ PCOS Consensus Workshop Group. Fertil Steril 2012; 97: 28-38.e25.

3. Brown J, Farquhar C, Beck J, Boothroyd C, Hughes E. Clomiphene, and anti-oestrogens for ovulation induction in PCOS. Cochrane Database Syst Rev 2009; (4): CD002249.
4. Balen AH. Ovulation induction in the management of anovulatory Polycystic Ovary Syndrome. Mol Cell Endocrinol 2013; 373: 77-82.

5. Van Santbrink EJ, Fauser BC. Ovulation induction in normogonadotropic anovulation (PCOS) Best Pract Res. Clin Endocrinol Metab 2006; 20: 261-70.

6. Homburg R, Hendriks ML, König TE, et al. Clomifene Citrate or low-dose FSH for the first-line treatment of infertile women with anovulation associated with Polycystic Ovary Syndrome: a prospective randomized multinational study. Hum Reprod 2012; 27: 468-473.

7. Amita M, Takahashi T, Tsutsumi S, et al. Molecular mechanism of the inhibition of estradiol-induced endometrial epithelial cell proliferation by Clomiphene Citrate. Endocrinology 2010; 151: 394-405.

8. Sereepapong W, Suwajanakorn S, Triratanachat S, et al. Effects of Clomiphene Citrate on the endometrium of regularly cycling women. Fertil Steril 2000; 73: 287-291.

9. Fetih AN, Habib DM, Abdelaal II, Hussein M, Fetih GN, Othman E R. Adding sildenafil vaginal gel to Clomiphene Citrate in infertile women with prior Clomiphene Citrate failure due to thin endometrium: a prospective self-controlled clinical trial. Facts Views Vis Obgyn 2017; 9: 21-27.

10. Hale SA, Jones CW, Osol G, Schonberg A, Badger GJ, Bernstein IM. Sildenafil increases uterine blood flow in nonpregnant nulliparous women. Reprod Sci 2010; 17: 358-365.

11. Richter KS, Bugge KR, Bromer JG, Levy MJ. Relationship between endometrial thickness and embryo implantation, based on 1,294 cycles of in vitro fertilization with transfer of two blastocyst-stage embryos. Fertil Steril 2007; 87: 53-59.

12. Rotterdam ESHRE/ASRM-Sponsored PCOS Consensus Workshop Group. Revised 2003 consensus on diagnostic criteria and long-term health risks related to Polycystic Ovary Syndrome. Fertil Steril 2004; 81: 19-25.

13. Teede HJ, Misso ML, Costello MF, et al. International PCOS Network. Recommendations from the international evidence-based guideline for the assessment and management of Polycystic Ovary Syndrome. Fertil Steril 2018; 110: 364-379.

14. Abdelazim IA, Amer OO, Farghali M. Common endocrine disorders associated with the Polycystic Ovary Syndrome. Menopause Rev 2020; 19: 179-183.

15. Abdelazim IA, Kanshaiym S. Abdelazim and Sakiyeva endocrinopathy associated with Polycystic Ovary Syndrome: case reports. J Family Med Prim Care 2019; 8: 3039-3041.

16. Abdelazim IA, Alanwar A, AbuFaza M, et al. Elevated and diagnostic androgens of Polycystic Ovary Syndrome. Menopause Rev 2020; 19: 1-5.

17. Fahmy AA, El Sokkary M, Sayed S. The value of oral Sildenafil in the treatment of female infertility: a randomized clinical trial. Life Sci J 2015; 12: 78-82.

18. Mohamed TY. Oral Sildenafil for treatment of female infertility among PCO patients: randomized comparative study. Austin J Obstet Gynecol 2019; 6: 114.

19. Check JH, Graziano V, Lee G, Nazari A, Choe JK, Dietterich C. Neither sildenafil nor vaginal estradiol improves endometrial thickness in women with thin endometria after taking oral estradiol in graduating dosages. Clin Exp Obstet Gynecol 2004; 31: 99-102.

20. Paulus WE, Strehler E, Zhang M, Jelinkova L, El-Danasouri I, Sterzik K. Benefit of Vaginal Sildenafil citrate in assisted reproductive therapy. Fertil Steril 2002; 77: 846-847.

21. Fisch P, Casper RF, Brown SE, et al. Unexplained infertility: evaluation of treatment with Clomiphene Citrate and human chorionic gonadotrophin. Fertil Steril 2003; 51: 828.

22. Sher G, Fisch JD. Vaginal Sildenafil (Viagra): a preliminary report of a novel method to improve uterine artery blood flow and endometrial development in patients undergoing IVF. Hum Reprod 2000; 15: 806-809.

23. Takasaki A, Tamura H, Miwa I, Taketani T, Shimamura K, Sugino N. Endometrial growth and uterine blood flow: a pilot study for improving endometrial thickness in the patients with a thin endometrium. Fertil Steril 2010; 93: 1851-1858.

24. Benni JM, Patil PA. An overview on sildenafil and female infertility. In J Health Sci 2016; 9: 131-136.

25. Das V, Agarwal A, Pandey A, Jain V, Agarwal S, Ara A. Evaluation of role of sildenafil in improving IUI success rates. Intern J Gynecol Obstet 2009; 107S2: S413-S729. 
26. Malinova $M$, Abouyta T, Krasteva $M$. The effect of vaginal sildenafil citrate on uterine blood flow and endometrium in the infertile women. Akush Ginekol (Sofiia) 2013; 52 (Suppl 1): 26-30.

27. Ashoush S, Abdelshafy A. Sildenafil citrate adjuvant treatment in women with polycystic ovary syndrome following clomiphene failure: a randomized controlled trial. EBWHJ 2019; 9: 487-493.

28. Li X, Luan T, Zhao C, et al. Effect of sildenafil citrate on treatment of infertility in women with a thin endometrium: a systematic review and meta-analysis. J Int Med Res 2020; 48: 300060520969584.

29. Gutarra Vilchez RB, Bonfill Cosp X, Glujovsky D, Viteri García A, Runzer Colmenares FM, Martinez Zapata MJ. Vasodilators for women undergoing fertility treatment. Cochrane Database Syst Rev 2018; 10: CD010001.

30. Berman JR, Laura AB, Steven MT, et al. Safety and efficacy of sildenafil citrate for the treatment of female sexual arousal disorder: a Doubleblind, placebo controlled study. J Urol 2003; 170: 2333-2338.

31. Basson R, Rosemery M, Mike DS, Gemma H, Naden K. Efficacy and safety of sildenafil citrate in women with sexual dysfunction associated with female sexual arousal disorder. J Womens Health Gend Based Med 2002; 11: 367-377. 